\title{
Nature of Capillary Condensation and Evaporation Processes in Ordered Porous Materials
}

\author{
Annie Grosman* and Camille Ortega* \\ Institut des Nanosciences de Paris (INSP), Universités Paris 6 et 7, UMR-CNRS 75-88, \\ Campus Boucicaut, 140 rue de Lourmel, 75015 Paris, France
}

Received April 18, 2005. In Final Form: July 27, 2005

\begin{abstract}
We report on a detailed experimental study of capillary condensation-evaporation processes of $\mathrm{N}_{2}$ in ordered mesoporous SBA-15 silica. We have carried out measurements of boundary hysteresis loops, reversal curves, and subloops in order to test whether this material behaves as an assembly of independent cylindrical pores open at both ends. With these data, we come to the conclusion that, whereas the boundary hysteresis loop has the classical shape of type $\mathrm{H} 1$ associated with condensation-evaporation in cylindrical pores open at both ends, the capillary evaporation does not take place at equilibrium as it is generally assumed. Moreover, the pores do not desorb independently of one another.
\end{abstract}

\section{Introduction}

Capillary condensation in mesoporous material is generally irreversible as revealed by adsorption experiments. ${ }^{1}$ The most studied hysteresis loops, named $\mathrm{H} 1$ and $\mathrm{H} 2$ in the IUPAC classification, ${ }^{2}$ reflect a widely accepted correlation between the shape of the hysteresis loop and the morphology of the mesoporous adsorbent.

The hysteresis loop of type $\mathrm{H} 1$ is characteristic of capillary condensation and evaporation processes in a cylindrical pore open at both ends. This irreversibility is considered as an intrinsic property of a fluid confined in a single pore related to the presence of metastable gaslike states, the evaporation proceeding through stable states via two meniscii formed at the pore ends. ${ }^{3,4}$ Most of the theoritical calculations corroborate this idea. ${ }^{5-11}$ Thus, for an assembly of independent cylindrical pores, a hysteresis loop with parallel adsorption and desorption branches is expected, the slope of which reflects the pore size distribution. ${ }^{12}$ From an experimental point of view, hysteresis loops of type $\mathrm{H} 1$ are observed in the case of mesoporous silicas MCM-41 and SBA-15, which exhibit a regular array of cylindrical pores. Therefore, it is generally admitted that the filling and draining of these porous materials proceed as described above. ${ }^{13,14}$

* To whom correspondence should be addressed. Telephone: (33) 1442746 44. Fax: (33) 1442728 78. E-mail: annie.grosman@ insp.jussieu.fr (A.G.); camille.ortega@insp.jussieu.fr (C.O.).

(1) Gregg, S. J.; Sing, K. S. W.Adsorption, Surface Area and Porosity; Academic Press: London, 1982; Chapter 3.

(2) Sing, K. S. W.; Everett, D. H.; Haul, R. A.; Moscou, L.; Pierotti,

R. A.; Rouquerol, J.; Siemieniewska, T. Pure Appl. Chem. 1985, 57, 603.

(3) Cohan, L. H. J. Am. Chem. Soc. 1938, 60, 433

(4) Everett, D. H. In The Solid Gas Interface; Flood, E. A., Ed.; Marcel

Dekker: New York, 1967; Vol. 2, Chapter 36, pp 1055-1113.

(5) Saam, W. F.; Cole, M. W. Phys. Rev. B 1975, 11, 1086.

(6) Evans, R.; Marini Bettolo Marconi, U.; Tarazona, P. J. Chem. Phys. 1986, 84, 2376.

(7) Papadopoulou, A.; Van Swol, F.; Marini Bettolo Marconi, U.; Tarazona, P. J. Chem. Phys. 1992, 97, 6942.

(8) Maddox, M. W.; Gubbins, K. E. Langmuir 1995, 11, 3988.

(9) Celestini, F. Phys. Rev. Lett. A 1997, 228, 84.

(10) Gelb, L. D.; Gubbins, K. E.; Radhakrishnan, R.; SliwinskaBartkowiak, M. Rep. Prog. Phys. 1999, 62, 1573.

(11) Gelb, L. D. Mol. Phys. 2002, 100, 2049.

(12) Ball, R. P.; Evans, R. Langmuir 1989, 5, 714

(13) Neimark, A. V.; Ravikovitch, P. I.; Vishnyakov, A. Phys. Rev. E 2000, 62, R1493.

(14) Ravikovitch, P. I.; Neimark, A. V. J. Phys. Chem. B 2001, 105, 6817.
Hysteresis loops of type $\mathrm{H} 2$ are generally found in disordered porous materials such as Vycor porous glass ${ }^{15}$ or, recently, in ordered mesoporous material with cagelike pores. ${ }^{16,17}$ The shape is asymmetrical with an evaporation branch steeper than the condensation branch. The most complete experimental data illustrating these hysteresis loops are those of Brown. ${ }^{15}$ Mason has developped a model ${ }^{18,19}$ to explain the family of adsorption/desorption isotherms obtained by Brown. In this model, a pore is connected to adjacent pores via constrictions and the evaporation of the liquid from this pore is blocked by the surrounding constrictions filled by the liquid (pore blocking effect). The constriction through which a pore may empty depends on whether the adjacent pores are full or empty. Thus, the pores do not empty independently of one another, and the interaction mechanism is pore blocking due to the connectivity (network effect).

Up until now, H1 and H2 hysteresis loops are thus considered as the signature of ordered porous material with cylindrical pores open at both ends and of disordered/ interconnected porous material, respectively.

In a recent adsorption study ${ }^{20}$ we report on the presence of hysteresis loops of type $\mathrm{H} 2$ in nitrogen adsorption isotherms obtained from $\mathrm{p}^{+}$-type porous silicon layers and membranes with pores open at one end or at both ends, respectively. It is a well ordered porous material with noninterconnected tubular pores perpendicular to the (100) Si substrate, separated from one another by singlecrystal walls of constant thickness. We have thus questioned ${ }^{20}$ the pore blocking effect as being the cause of the observed irreversibility. We came to the conclusion that the condensation branch for the porous layers with pores closed at one end represents the thermodynamical equilibrium for the two porous systems (porous layer and membrane), the evaporation proceeding through metastable states in both configurations. We will come back more fully to the details of these experiments below. Since these results, another experimental study, carried out with

(15) Brown, A. J., Thesis, Bristol, 1963.

(16) Ravikovitch, P. I.; Neimark, A. V. Langmuir 2002, 18, 9830.

(17) Morishige, K.; Tateishi, N. J. Chem. Phys 2003, 119, 2301.

(18) Mason, J. Proc. R. Soc. London 1983, A 390, 47.

(19) Mason, J. Proc. R. Soc. London 1988, A 415, 453.

(20) Coasne, B.; Grosman, A.; Ortega, C.; Simon, M. Phys. Rev. Lett. 2002, 88, 256102. 


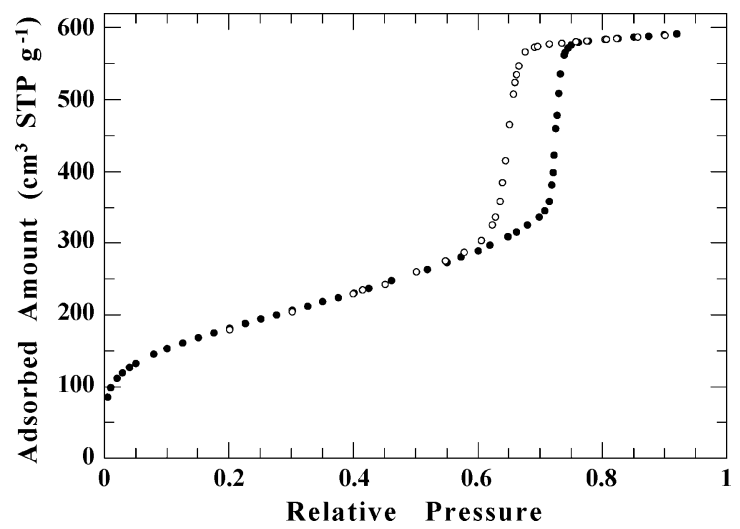

Figure 1. Nitrogen adsorption isotherm for the SBA-15 silica at $77.4 \mathrm{~K}$.

$\mathrm{p}^{+}$-type porous silicon, has also raised questions about pore blocking effect. ${ }^{21}$

Question then arises about the existence of such metastable liquidlike states in other ordered porous systems and in particular in MCM-41 and SBA-15 mesoporous silicas.

Our idea was the following. If actually the draining in MCM-41 and SBA-15 proceeds through stable states, as it is generally admitted, each pore should drain independently of the others and the hysteretic behavior should be described by the Preisach model ${ }^{22}$ originated in studies of the hysteretic behavior of magnetic systems and adapted to adsorption in porous materials by Everett ${ }^{4}$ in his independent-domain theory. This has motivated the present detailed study of capillary condensation-evaporation in SBA-15 material. We present and discuss measurements of global hysteresis loop, reversal scanning curves, and subloops.

\section{Experimental Section}

Measurements. The adsorption-desorption isotherms, the primary descending scanning curves, and the subloops, carried out with nitrogen at $77.4 \mathrm{~K}$, were measured using a Micromeritics ASAP 2010 instrument. The SBA-15 silica sample was outgassed at $120{ }^{\circ} \mathrm{C}$ for $6 \mathrm{~h}$ and then at ambient temperature for 1 day.

Materials. The mesoporous silica SBA-15 under study has been synthesized by Morineau and co-workers ${ }^{23}$ according to the procedure reported by Zhao and co-workers. ${ }^{24}$ Triblock copolymer Pluronic P123 (EO20PO70EO20) was used as surfactant and tetraethoxylsilane (TEOS) as silica source. The chemical composition of the reaction mixture (copolymer, TEOS, $\mathrm{H}_{2} \mathrm{O}, \mathrm{HCl}$ ) was the same as that used by Zhao and co-workers. ${ }^{24}$ After reaction at $35{ }^{\circ} \mathrm{C}$ for $20 \mathrm{~h}$, followed by synthesis at $100{ }^{\circ} \mathrm{C}$ for a further $24 \mathrm{~h}$, the mixture was collected by filtration without washing, dried, and calcined in air at $500{ }^{\circ} \mathrm{C}$ for $6 \mathrm{~h}$. The structure factor of the sample, measured by neutron scattering experiments, ${ }^{23}$ exhibits Bragg peaks due to the honeycomb-type arrangement of the cylindrical pores. The intense (100) peak reflects a $d$ spacing of $9.094 \mathrm{~nm}$ corresponding to a unit cell parameter $a=10.5 \mathrm{~nm}$.

Figure 1 shows the nitrogen adsorption-desorption isotherm at $77.4 \mathrm{~K}$. The position of the hysteresis loop on the pressure axis is very similar to those observed by Kruk and co-workers (sample S4-C in Figure 4) ${ }^{25}$ and Jun and co-workers. ${ }^{26}$

(21) Wallacher, D.; Künzner, N.; Kovalev, D.; Knorr, N.; Knorr, K. Phys. Rev. Lett. 2004, 92, 195704.

(22) Preisach, F. Z. Phys. 1935, 94, 277.

(23) Morineau, D.; Xia, Y.; Alba-Simionesco, C. J. Chem. Phys. 2002, 117,8966

(24) Zhao, D.; Feng, J.; Hui, Q.; Melosh, N.; Fredrickson, G.; Smelka,

B.; Stucky, G. D. Science 1998, 279, 548.

(25) Kruk, M.; Jaroniec, M.; Ko, C. H.; Ryoo, R. Chem. Mater. 2000, 12,1961

(26) Jun, S.; Joo, S. H.; Ryoo, R.; Kruk, M.; Jaroniec, M.; Liu, Z.; Ohsuna, T.; Terasaki, O. J. Am. Soc. 2000, 122, 10712.
The isotherm presents a typical hysteresis loop of type H1 with steep condensation and evaporation branches. The steepness of the condensation branch $\left(0.71<p=P / P_{0}<0.75\right)$ is indicative of a narrow mesopore size distribution and characteristic of good quality material. At the end of the pore filling, we can observe a further increase of the adsorbed amount due to adsorption on the external surface $\left(S_{\text {ext }}\right)$ of the porous material. The specific surface area $\left(S_{\mathrm{BET}}\right)$ and the $C$ factor, calculated using the Brunauer-Emmet-Teller ${ }^{27}$ method (BET) in a relative pressure range from 0.04 to 0.2 , are equal to $650 \mathrm{~m}^{2} / \mathrm{g}$ and to 120 , respectively.

Several studies $25,26,28-31$ indicate that the structure of SBA-15 silica is more complex than an array of hexagonally ordered cylindrical pores and exhibits, in addition to the uniform mesopores, a significant number of disordered micropores located in the walls separating the mesopores. These micropores are suggested to form as a result of the penetration of the poly(ethylene oxide) chains of the triblock copolymer template in the silica framework. A detailed discussion is given by Ryoo and co-workers ${ }^{31}$ related to the origin of these complementary pores the size of which was estimated to be down to $\approx 3 \sim 4 \mathrm{~nm}$.

The micropore volume and the total pore volume are usually estimated using the $\alpha_{\mathrm{s}}$-plot or the $\beta$-plot method. ${ }^{1}$ The $\alpha_{\mathrm{s}}$-plot method consists of plotting the amount adsorbed in the porous material under study, $V(p)$, as a function of a standard reduced adsorption $\alpha_{s}(p)=V_{\text {ref }}(p) / V_{\text {ref }}(0.4)$, where $V_{\text {ref }}(p)$ and $V_{\text {ref }}(0.4)$ are the amount adsorbed on the reference material at relative pressures $p$ and 0.4 , respectively. In the case where both solids proceed via the same mechanism, that is via multilayer adsorption, and if the variation of the surface area of the porous material as a function of the adsorbed amount is negligible as in a nonporous medium, the $\alpha_{\mathrm{s}}$-plot is linear from the beginning of the adsorption to the pressure at which capillary condensation occurs in the mesopores. When the porous material contains micropores, the initial part of the $\alpha_{s}$-plot exhibits a sharp increase corresponding to the emptying of the micropores and then the $\alpha_{s}$-plot grows softer when the micropores are filled with condensed adsorbate. The intercept on the adsorption axis of the extrapolated linear branch, after the micropores are filled, when converted to a liquid volume, is generally taken as equal to the micropore volume. When the pore volume is filled, the total adsorbed amount $V_{\mathrm{f}}(p)=V_{\mathrm{p}}+\eta \alpha_{\mathrm{s}}(p)$ can be used to estimate the pore volume $V_{\mathrm{p}}$. The standard reduced nitrogen adsorption isotherm at $77 \mathrm{~K}$, $\alpha_{\mathrm{s}}(p)$, for a macroporous material (LiChrospher Si-1000 silica), has been extracted from the data reported by Jaroniec and coworkers. ${ }^{32}$

In the $\beta$-plot method, $V(p)$ is represented as a function of the statistical film thickness corresponding to the reference material.

Figure 2 shows the $\alpha_{\mathrm{s}}$-plot corresponding to the SBA-15 sample under study.

The initial part of the $\alpha_{\mathrm{s}}$-plot is linear and passes through the origin of the graph. Thus, for the SBA-15 silica under study, the micropore volume cannot be detected by this method. Similar results have been obtained by other groups ${ }^{29-31}$ for samples formed in similar conditions (synthesized temperature: $100^{\circ} \mathrm{C}$ ). The pore volume $V_{\mathrm{p}}$, deduced from the relation $V_{\mathrm{f}}(p)=V_{\mathrm{p}}+$ $\eta \alpha_{\mathrm{s}}(p)$, is equal to $565 \mathrm{~cm}^{3} \mathrm{STP} / \mathrm{g}=0.87 \mathrm{~cm}^{3} / \mathrm{g}$. The value of the mesopore diameter $D$ can be estimated through the following relation:

$$
D=c d\left[\rho V_{\mathrm{mp}} /\left(1+\rho V_{\mathrm{p}}\right)\right]^{1 / 2}
$$

where $c=1.213$ is a geometrical factor, $d$ the interplanar spacing, $\rho$ the pore wall density assumed to be equal to that of amorphous silica $\left(2.2 \mathrm{~g} / \mathrm{cm}^{3}\right)$, and $V_{\mathrm{mp}}$ the mesopore volume. Assuming $V_{\mathrm{mp}}$

(27) Brunauer, S.; Emmett, P. H.; Teller, E. J. Am. Chem. Soc. 1938 60,309

(28) Lukens, W. W. Jr.; Schmidt-Winkel, P.; Zhao, D.; Feng, J.; Stucky, G. D. Langmuir 1999, 15, 5403.

(29) Imperor-Clerc, M.; Davidson, P.; Davidson, A. J. Am. Chem. Soc. 2000, 122, 11925

(30) Galarneau, A.; Cambon, H.; Di Renzo, F.; Fajula, F. Langmuir 2001, 17, 8328

(31) Ryoo, R.; Ko, C. H.; Kruk, M.; Antochshuk, V.; Jaroniec, M. J. Phys. Chem. B 2000, 104, 11465.

(32) Jaroniec, M.; Kruk, M.; Olivier, J. P. Langmuir 1999, 15, 5410. 


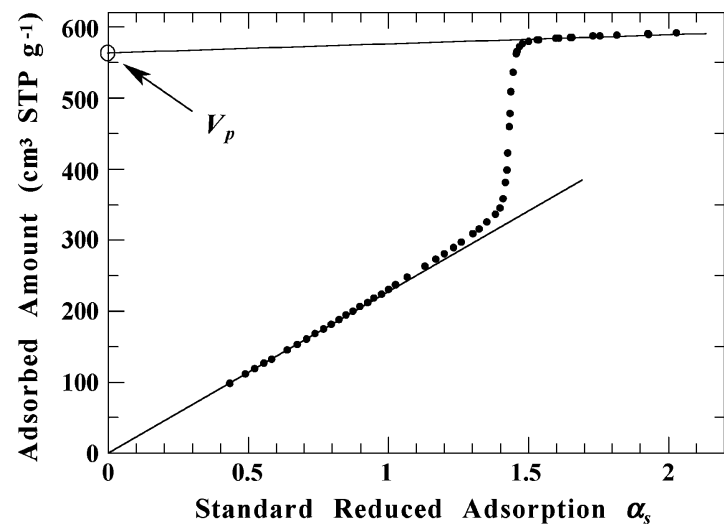

Figure 2. $\alpha_{s}$-plot for the SBA-15 silica. The reference material data, $\alpha_{s}(p)$, is for macroporous LiChrospher Si-1000 silica. ${ }^{34}$

Table 1. Main Structural Parameters of SBA-15 Silicas ${ }^{a}$

\begin{tabular}{lccllc}
\hline \multicolumn{1}{c}{ sample } & $\begin{array}{c}a \\
(\mathrm{~nm})\end{array}$ & $\begin{array}{c}V_{\mathrm{p}} \\
\left(\mathrm{cm}^{3} / \mathrm{g}\right)\end{array}$ & $\begin{array}{c}V_{\mu \mathrm{p}} \\
\left(\mathrm{cm}^{3} / \mathrm{g}\right)\end{array}$ & $\begin{array}{c}D_{\max } \\
(\mathrm{nm})\end{array}$ & $\begin{array}{c}S_{\mathrm{BET}} \\
\left(\mathrm{m}^{2} / \mathrm{g}\right)\end{array}$ \\
\hline this study & $10.5^{b}$ & $0.87^{c}$ & $*$ & 8.95 & 650 \\
S4-C. ref 25 & $10.6^{a}$ & $0.92^{c}$ & 0.06 & 9.1 & 850 \\
P123BC. ref 29 & $11.05^{a}$ & $1.06^{d}$ & $*$ & 9.7 & 840 \\
ref 30 & $10.5^{a}$ & $1^{d}$ & $*$ & 9.15 & 750
\end{tabular}

${ }^{a} a$, unit cell parameter determined by $\mathrm{XRD}^{a}$ or by neutron scattering experiment ${ }^{b} ; V_{\mathrm{p}}$, pore volume evaluated using the $\alpha_{\mathrm{s}}$ plot $^{c}$ or the $\beta$-plot ${ }^{d}$ method; $V_{\mu \mathrm{p}}$, micropore volume assessed using the $\alpha_{\mathrm{s}}$-plot or the $\beta$-plot method (* undetected by these methods); $D_{\max }$, mesopore diameter calculated on the basis of eq 1 with $V_{\mathrm{mp}}$ $=V_{\mathrm{p}}\left(V_{\mathrm{p}}\right.$ includes a contribution from the micropore volume, so the actual mesopore diameter is smaller); $S_{\mathrm{BET}}$, specific surface area, calculated using the usual $\mathrm{N}_{2}$ cross-section area: $0.162 \mathrm{~nm}^{2}$. For ref 30 , the parameters correspond to the sample synthesized at 100 ${ }^{\circ} \mathrm{C}$.

$=V_{\mathrm{p}}$, one obtains the maximum value of the mesopore diameter $D_{\max }=8.95 \mathrm{~nm}$. The mesopore surface area $S_{\mathrm{mp}}$ can be estimated through the relation $S_{\mathrm{mp}}=4 V_{\mathrm{mp}} / D_{\max }=390 \mathrm{~m}^{2} / \mathrm{g}$. Such a deviation with regards to $S_{\mathrm{BET}}=650 \mathrm{~m}^{2} / \mathrm{g}$ can be only explained by the presence of very small structures, the intrawall micropores. Assuming that the micropore volume $V_{\mu \mathrm{p}}$ is equal to $10 \%$ of the total pore volume, which seems to us a reasonable value, one finds $D=8.5 \mathrm{~nm}$ and $S_{\mathrm{mp}}=370 \mathrm{~m}^{2} / \mathrm{g}$. We think that the actual value of the mesopore diameter is not very far from $8.5 \mathrm{~nm}$. From the micropore surface area, $S_{\mu \mathrm{p}}=280 \mathrm{~m}^{2} / \mathrm{g}$, one can estimate the micropore size $D_{\mu \mathrm{p}}=4 V_{\mu \mathrm{p}} / S_{\mu \mathrm{p}}=1.2 \mathrm{~nm}$. Table 1 summarizes the main structural parameters corresponding to the sample under study and to samples made in a similar way by other groups. ${ }^{25,29,30}$

\section{Results}

First let us test the scenario for condensation and evaporation which defines the hysteresis loops of type $\mathrm{H} 1$ that is if SBA-15 behaves as an assembly of independent cylindrical pores. During adsorption, a film is formed on the pore walls which acquires a critical radius of curvature resulting in capillary condensation in the pore at a relative pressure $\alpha=P / P_{0}$ which is an increasing function of the pore radius $R$.

Evaporation from the pore occurs when the vapor pressure is lowered to the equilibrium pressure $\beta$ corresponding to a meniscus formed at both ends. In these conditions, a primary descending scanning curve (PDSC), initiated at a reversal point $A(\alpha)$ on the primary condensation branch, should result in the reversible desorption of the pores still empty at $A(\alpha)$ until it meets the boundary desorption branch at a relative pressure $\beta$, defining a pair $(\alpha, \beta)$ which characterizes a pore (Figure 3 ).

Figure 4 shows the boundary hysteresis loop and an experimental PDSC initiated at point $\mathrm{A}_{1}$ on the boundary condensation branch. The experimental PDSC does not

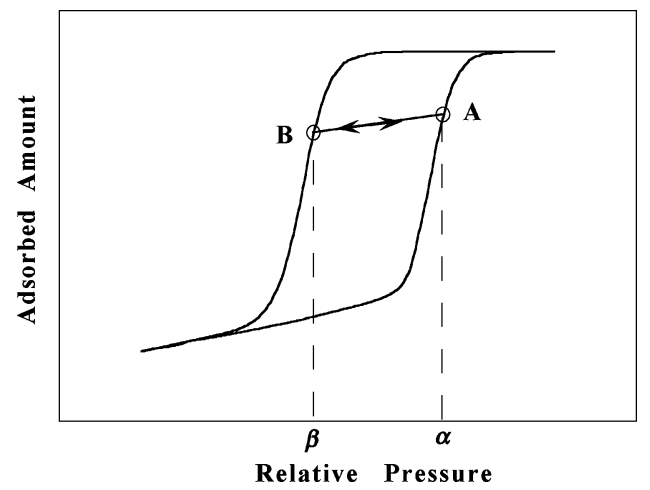

Figure 3. Schematic representation of a hysteresis loop of type $\mathrm{H} 1$ with a primary reversal curve $A B$. The process $A B$ corresponds to the reversible desorption of the pores still empty at point $\mathrm{A}$.

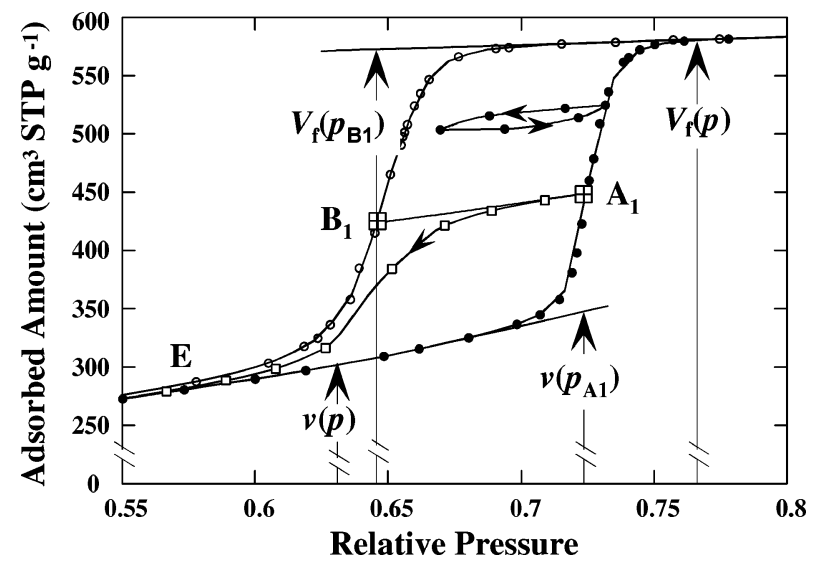

Figure 4. Hysteresis loop shown in Figure 1 with a primary descending scanning curve (PDSC) initiated at $\mathrm{A}_{1}$ (line with square symbol) and a subloop. The line without symbol, $\mathrm{A}_{1} \mathrm{~B}_{1}$, is the PDSC calculated according to eq 8. Also shown are the parameters used in the calculation of the PDSC: $V_{\mathrm{f}}(p)$ and $v(p)$ are the adsorbed amounts when the mesopores are filled and empty with a film on the pore walls, respectively. $V_{\mathrm{f}}\left(p_{\mathrm{B} 1}\right)$ and $v\left(p_{\mathrm{A} 1}\right)$ are their extreme extrapolated values.

meet the boundary desorption branch before the closure point E. Similar results have been obtained in MCM- $41^{33}$ and in SBA- $15^{34}$ silicas.

To quantify the difference between the experimental PDSC and the model, we have calculated a PDSC under the assumption that no evaporation occurs from the filled pores but only desorption from the pores still empty at point $\mathrm{A}_{1}$. The adsorbed amount along the $\operatorname{PDSC}, V_{\mathrm{PDSC}}(p)$, as a function of the relative pressure $p=P / P_{0}$, is represented by the following relation:

$$
\begin{aligned}
V_{\mathrm{PDSC}}(p)=V_{\mu \mathrm{p}}+S_{\mathrm{ext}} t(p)+ & (L-l) \pi R^{2}+ \\
& \quad l \pi\left\{R^{2}-[R-t(p)]^{2}\right\}
\end{aligned}
$$

where the first term represents the amount adsorbed in the intrawall micropores (the micropores are filled at lower pressure than the capillary condensation and evaporation pressures within the mesopores), the second term, the amount adsorbed on the external surface $S_{\text {ext }}(t(p)$ is the film thickness at pressure $p$ ), the third term, the volume of the mesopores filled at point $\mathrm{A}_{1}$ ( $L$ is the total length of the mesopores, $l$ the total length of the mesopores still

(33) McNall, M.; Laurence, R. L.; Conner, C. W. Microporous Mesoporous Mater. 2001, 44-45, 709 .

(34) Esparza, J. M.; Ojeda, M. L.; Campero, A.; Dominguez, A.; Rojas, F.; Vidales, A. M.; Lopez, R. H.; Zgrablich, G. Colloids Surf. A: Physicochem. Eng. Aspects 2004, 241, 35. 
empty at $\mathrm{A}_{1}$, and $R$ the mesopore mean radius), the last term being the amount adsorbed in the mesopores still empty at point $\mathrm{A}_{1}$.

Relation (2) may be written in a more suitable form

$$
\begin{array}{r}
V_{\mathrm{PDSC}}(p)=V_{\mu \mathrm{p}}+S_{\mathrm{ext}} t(p)+L \pi R^{2}-l \pi[R-t(p)]^{2}= \\
V_{\mathrm{f}}(p)-l \pi[R-t(p)]^{2}
\end{array}
$$

where $V_{\mathrm{f}}(p)$ is the adsorbed amount when the mesopores are filled (see Figure 4).

It is interesting to introduce $v(p)$, the amount of gas adsorbed before capillary condensation occurs in the mesopores $(p<0.70)$, through the following equation:

$$
\begin{aligned}
& v(p)=V_{\mu \mathrm{p}}+S_{\mathrm{ext}} t(p)+L \pi\{\left.R^{2}-[R-t(p)]^{2}\right\}= \\
& V_{\mathrm{f}}(p)-L \pi[R-t(p)]^{2}
\end{aligned}
$$

The ratio $l / L$, which represents the fraction of the mesopores still empty at point $A_{1}$, can be calculated through the experimental initial conditions

$$
V_{\mathrm{PDSC}}\left(p_{\mathrm{A} 1}\right)=V_{\mathrm{A} 1}=V_{\mathrm{f}}\left(p_{\mathrm{A} 1}\right)-l \pi\left[R-t\left(p_{\mathrm{A} 1}\right)\right]^{2}
$$

where $V_{\mathrm{A} 1}$ is the adsorbed amount at point $\mathrm{A}_{1}$ and through the extrapolated value

$$
v\left(p_{\mathrm{A} 1}\right)=V_{\mathrm{f}}\left(p_{\mathrm{A} 1}\right)-L \pi\left[R-t\left(p_{\mathrm{A} 1}\right)\right]^{2}
$$

calculated using an interpolation polynomial which fits the experimental data $v(p)$, between the relative pressures 0.2 and 0.68 .

We then obtain

$$
l / L=\left[V_{\mathrm{f}}\left(p_{\mathrm{A} 1}\right)-V_{\mathrm{A} 1}\right] /\left[V_{\mathrm{f}}\left(p_{\mathrm{A} 1}\right)-v\left(p_{\mathrm{A} 1}\right)\right]
$$

In the present experimental conditions $\left(p_{\mathrm{A} 1}=0.725\right)$, the ratio $l / L$ is equal to 0.58 .

Finally, from eqs 3,4 , and $7, V_{\mathrm{PDSC}}(p)$ can be expressed as a function of the experimental data $V_{\mathrm{f}}(p), v(p)$ and their extrapolated values (see Figure 4 ) to the exclusion of the unknown parameters such as the micropore volume $V_{\mu \mathrm{p}}$, the mean mesopore radius $R$, and the film thickness $t(p)$

$$
\begin{array}{r}
V_{\mathrm{PDSC}}(p)=V_{\mathrm{f}}(p)-(l / L)\left[V_{\mathrm{f}}(p)-v(p)\right]= \\
{[1-(l / L)] V_{\mathrm{f}}(p)+(l / L) v(p)}
\end{array}
$$

As the variations of $V_{\mathrm{f}}(p)$ are small compared to those of $v(p), V_{\mathrm{PDSC}}(p)$ varies proportionately to $v(p)$ as expected. The calculated PDSC is shown in Figure 4. The fact that the calculated PDSC is tangent to the experimental one in the beginning of the desorption process indicates that the extrapolated value $v\left(p_{\mathrm{A} 1}\right)$ is correctly estimated. An interpolation polynomial which fits the experimental data including higher pressures, $p=0.70$ for example, leads to a PDSC which passes slightly under the first experimental point after $\mathrm{A}_{1}$.

At pressure $p_{\mathrm{B} 1}$, the experimental PDSC is well below the calculated one which shows that evaporation occurs from a significant proportion of the filled pores in the course of the experimental PDSC process. This is confirmed by the presence of the subloop within the boundary hysteresis loop shown in Figure 4.

Thus, although the experimental boundary hysteresis loop has a shape which can be classified as type $\mathrm{H} 1$, the scenario for capillary condensation and evaporaton underlying a hysteresis loop of type $\mathrm{H} 1$ is not applicable.

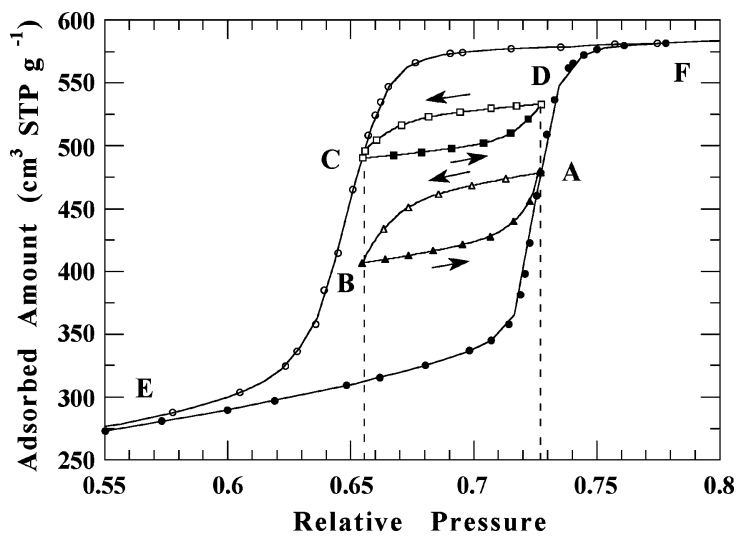

Figure 5. Hysteresis loop shown in Figure 1 with two subloops measured between the same pressure end points $p_{\mathrm{A}}$ and $p_{\mathrm{B}}$.

The comparison between the experimental PDSC and the boundary evaporation branch suggests that the evaporation pressure of a pore depends on whether the porous system is completely filled or not, that is, that the pores in SBA-15 do not drain independently of one another. Pore blocking/network effects are generally invoked to explain such PDSC. ${ }^{33}$

Esparza and co-workers have obtained similar PDSCs in SBA-15 (Figure 7, sample SBA-15-3). ${ }^{34}$ On the basis of the analysis of experimental PDSCs by means of nonlocal density functional theory, they explain their results in a different way. The mesopores are not perfectly cylindrical but exhibit modulations in their diameter. During the desorption process there is "a sort of pore blocking effect that occurs in cylindrical tubes of varying cross-section: instead of following an ideal desorption mechanism in which a pore of a given diameter evaporates completely its condensate at a particular relative pressure, the emptying of pore entities in real SBA-15 substrates occurs more gradually". Indeed, this could explain that evaporation occurs along a primary descending curve as shown by the experimental result of Figure 4, but, according to the mechanism proposed by Esparza and co-workers, ${ }^{34}$ the pores should empty independently of one another.

To decide between these two interpretations, interaction between the pores or "single-pore blocking effect", it is necessary to use an unambiguous test of the independence of the pores that is the comparison of two subloops such as that shown in Figure 5, initiated, the one on the primary filling branch, the other on the primary draining branch, between the same relative pressure end points., ${ }^{4,2}$

For a system composed of independent pores, these two subloops should have an identical shape or in other words should be congruent. It must be noted that this test can be rigorously applied only to systems which can be treated as an assembly of two-state microsystems which is not the case for adsorption in porous materials. Indeed, a pore is not either full or empty but full or empty with a film on the pore walls, the thickness of which cannot be a priori neglected. Along the two subloops, the state of the system is not exactly the same, the number of empty pores being higher for the lower subloop than for the higher. Thus, the amount of gas desorbed from the empty pores is higher along the path $\mathrm{AB}$ than along the path DC.

To illustrate the state of the system along these two subloops, it is useful to use the Preisach model. ${ }^{22}$ In this model, adapted to capillary condensation in porous materials by Everett, ${ }^{4}$ a domain (a pore in our case) is represented by a pair $(\alpha, \beta)$ in the $\alpha-\beta$ plane where $\alpha$ and $\beta$ are the condensation and evaporation pressures, re- 

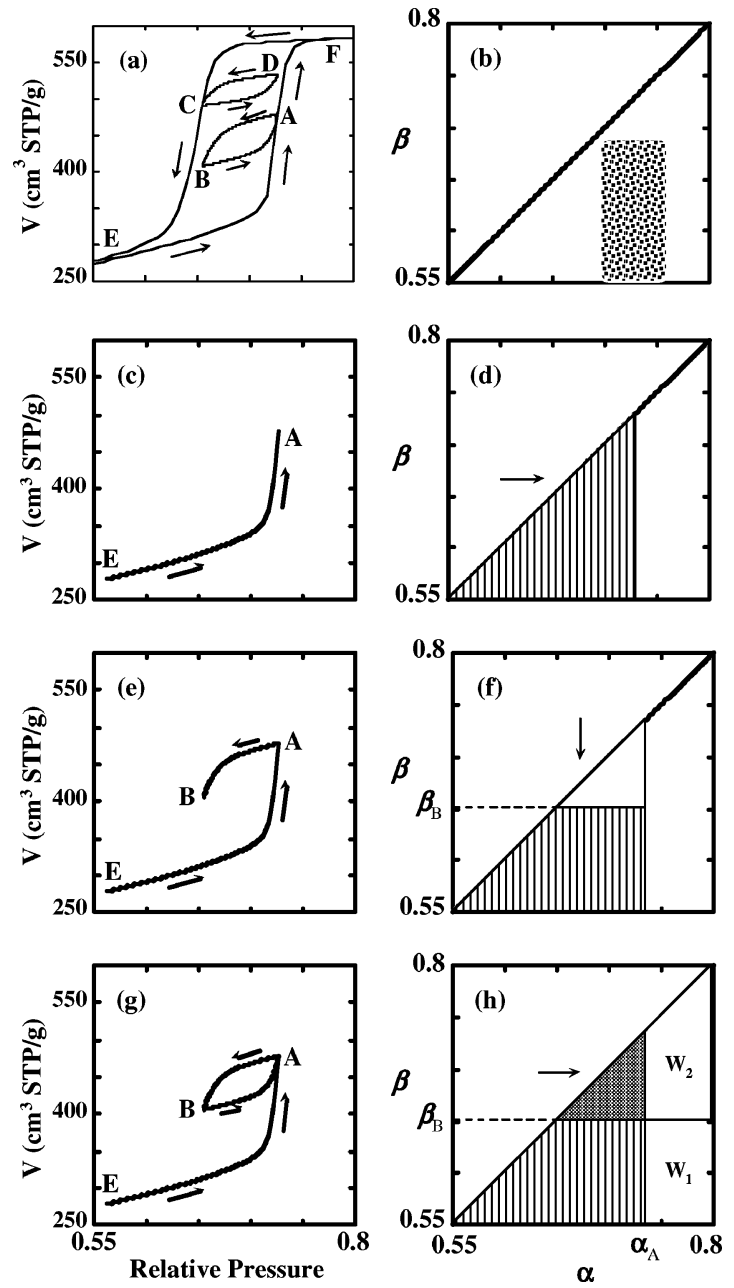

Figure 6. Illustration of condensation-evaporation processes, along the cycle ABA, using the Preisach model. In panel a are shown the boundary hysteresis loop and the subloops ABA and CDC. In panel $b$, a collection of pores is schematically mapped onto the $\alpha-\beta$ space, each pore being represented by a single point. The vertical shading lines in panels $d$ and $f$ indicate which set of pores is filled at points A and B, respectively. In panel $h$, the vertical shading lines indicate which set of pores remains filled and shaded triangle which set of pores fills and drains along the cycle $\mathrm{AB}$. In all cases, the white regions $\mathrm{W}_{1}$ and $\mathrm{W}_{2}$ represent the empty pores. The white region $\mathrm{W}_{1}$ indicates which set of pores remains empty along the cycle ABA but filled along the cycle CDC, see Figure 7.

spectively. As $\beta<\alpha$, the pairs $(\alpha, \beta)$ are located on a triangular region below the diagonal $\alpha=\beta$. In the independent-domain theory, the pairs $(\alpha, \beta)$ are independent of the state of the system.

The state of the system during the processes leading to EA and EFC and during the cycles (ABA) and (CDC) is represented by diagrams such as Figure 6 and Figure 7. In each figure, we have represented the condensationevaporation processes on the left and the corresponding Preisach diagram on the right.

In Figure 6a are shown the boundary hysteresis loop and the two subloops ABA and CDC. In Figure 6b, all pores in the system are schematically represented by points in a rectangular region defined by $0.7<\alpha<0.76$ and $0.55<\beta<0.69$. In Figure 6 , panels $d$ and $\mathrm{f}$, the vertical shading lines represent the pores that fill along the path EA and the pores that remain filled after the desorption process $A B$, respectively. Figure $6 \mathrm{~h}$ represents the three different regions which can be distinguished along the cycle ABA: the vertical shading lines represent the pores
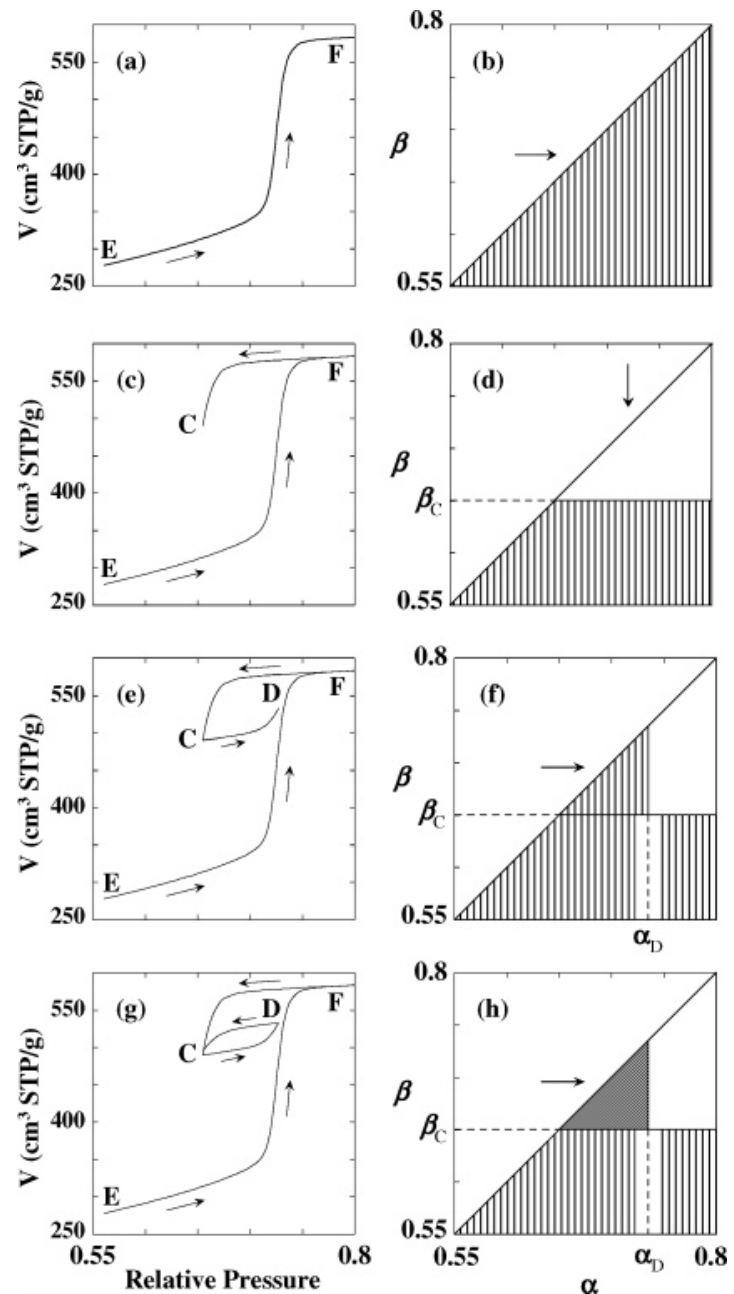

Figure 7. Illustration of condensation-evaporation processes, along the cycle CDC, using the Preisach model, as in Figure 6.

which remain filled, the shaded triangle the pores which empty (along $\mathrm{AB}$ ) and fill (along $\mathrm{BA}$ ) and the white regions, $\mathrm{W}_{1}$ and $\mathrm{W}_{2}$, the pores that remain empty with a film on the pore walls, the thickness of which varies along the cycle (ABA).

Figure 7 corresponds to the subloop CDC. On comparing Figures $6 \mathrm{~h}$ and $7 \mathrm{~h}$, we can see that the same pores fill and empty during the two cycles (shaded triangle), but as noted above, the state of the system is different along the two cycles: the pores represented by the white region $\mathrm{W}_{1}$ in Figure $6 \mathrm{~h}$ remain empty during the cycle ABA, whereas they remain filled during the cycle CDC. Thus, to compare the two subloops, we have to take into account the amount of gas desorbed along the path $\mathrm{AB}$ from the pores represented by the white region $\mathrm{W}_{1}$.

The total length $\left(l^{\prime}\right)$ of these pores can be expressed as a function of the difference $V_{\mathrm{D}}-V_{\mathrm{A}}$ through the formula

$$
\begin{aligned}
& V_{\mathrm{D}}-V_{\mathrm{A}}=l^{\prime} \pi R^{2}-l^{\prime} \pi\left\{R^{2}-\left[R-t\left(p_{\mathrm{A}}\right)\right]^{2}\right\}= \\
& l^{\prime} \pi\left[R-t\left(p_{\mathrm{A}}\right)\right]^{2}
\end{aligned}
$$

where $V_{\mathrm{D}}$ and $V_{\mathrm{A}}$ are the adsorbed amount at points D and $A$ (see Figures $6 \mathrm{~d}$ and $7 \mathrm{f}$ which represent the state of the system at $\mathrm{A}$ and $\mathrm{D}$, respectively).

$\Delta(p)$, the amount of gas desorbed from these pores along the path $\mathrm{AB}$, is 


$$
\begin{array}{r}
\Delta(p)=l^{\prime} \pi\left\{\left[R^{2}-\left(R-t\left(p_{\mathrm{A}}\right)\right)^{2}\right]-\left[R^{2}-(R-t(p))^{2}\right]\right\}= \\
l^{\prime} \pi\left\{[R-t(p)]^{2}-\left[R-t\left(p_{\mathrm{A}}\right)\right]^{2}\right\} \quad(10)
\end{array}
$$

From eqs 9 and 10, we get

$$
\Delta(p)=\left(V_{\mathrm{D}}-V_{\mathrm{A}}\right)\left\{\left[(R-t(p))^{2} /\left(R-t\left(p_{\mathrm{A}}\right)\right)^{2}\right]-1\right\}
$$

Using eqs 4 and 7 , we can determine the ratio $[R-t(p)]^{2 /}$ $\left[R-t\left(p_{\mathrm{A}}\right)\right]^{2}$ as a function of the experimental data $V_{\mathrm{f}}(p)$ and $v(p)$ and their extrapolated values

$$
\begin{aligned}
& {[R-t(p)]^{2} /\left[R-t\left(p_{\mathrm{A}}\right)\right]^{2}=} \\
& \quad\left[V_{\mathrm{f}}(p)-v(p)\right] /\left[V_{\mathrm{f}}\left(p_{\mathrm{A}}\right)-v\left(p_{\mathrm{A}}\right)\right]
\end{aligned}
$$

Equation 11 becomes

$\Delta(p)=\left(V_{\mathrm{D}}-V_{\mathrm{A}}\right)\left\{\left[\left(V_{\mathrm{f}}(p)-v(p)\right) /\left(V_{\mathrm{f}}\left(p_{\mathrm{A}}\right)-v\left(p_{\mathrm{A}}\right)\right)\right]-1\right\}$

Before comparing the two subloops ABA and CDC, we have to add $\Delta(p)$ to the adsorbed amount along the cycle (ABA). The two subloops and the corrected cycle AB'A are represented in Figure 8 where the right end point $D$ of the cycle CDC is offset to coincide with $\mathrm{A}$.

The subloops (CDC) and ( $\left.\mathrm{AB}^{\prime} \mathrm{A}\right)$ are not congruent which confirms that the mesopores in SBA-15 material do not drain independently of one another.

\section{Discussion}

The present results indicate that the open-ended mesopores of SBA-15 do not behave as an assembly of independent pores with regards to the evaporation process. When the system is completely filled, the evaporation pressure of the whole of the pores is delayed, whereas when it is not completely filled, a part of the system can evaporate at higher pressure. The interaction mechanism generally invoked to explain such a behavior is pore blocking/network effects. According to the model developped by Mason, ${ }^{18,19}$ pore blocking/network effects can be observed provided the porous material is composed of pores with variable sizes connected to one another via constrictions. Let us analyze the relevance of pore blocking/ network effects in SBA-15.

(i) Interconnections between the Mesopores (3D Network). In SBA-15, the mesopores are connected by intrawall micropores, the presence of which has been explained by the partial occlusion of the poly(ethylen oxide) chains into the silica matrix which becomes microporous upon calcination. According to Ryoo and co-workers, ${ }^{31}$ the fact that the initial part of the $\alpha_{\mathrm{s}}$-plots does not exhibit any step, as is the case in the SBA-15 under study, is indicative of a broad pore size distribution in the micropore-small mesopore range. However, as the closure point of the hysteresis loops ( 0.55 in our case) is generally well above $P / P_{0}=0.4$, which is the lower limit of adsorption-desorption hysteresis on nitrogen isotherm at $77 \mathrm{~K}$, the interconnection size distribution does not extend to the primary mesopores. The size of the intrawall pores is therefore lower than the size of the mesopores and has been estimated to be below $3 \sim 4 \mathrm{~nm}^{31}$ so that they remain full at pressures when capillary condensation and evaporation occur in the mesopores and do not play any role in the condensation-evaporation processes in the mesopores.

(ii) Constrictions within the Mesopores (1D Network). What is known about the mesopore morphology in SBA15 ? The presence of intrawall micropores certainly induces

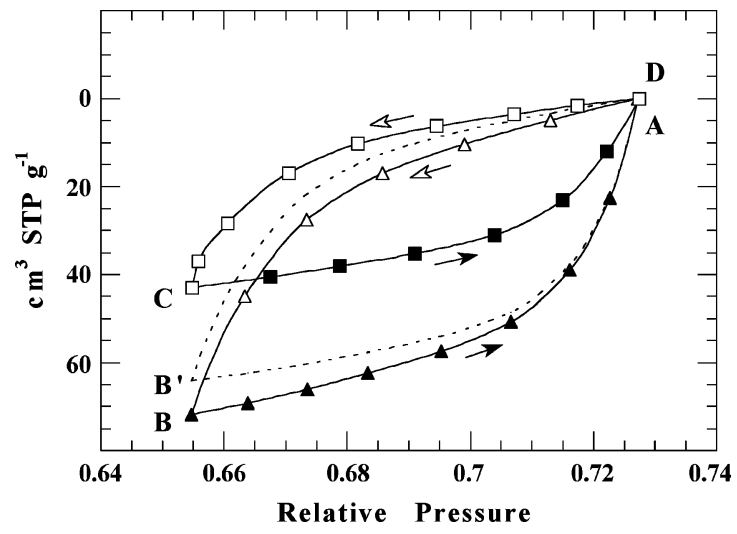

Figure 8. Comparison of the subloops shown in Figure 5. The right end points $A$ and $D$ are offset to overlap. The dash line $\mathrm{AB}^{\prime} \mathrm{A}$ represents the subloop $\mathrm{ABA}$ after correction according to eq 13. $\Delta\left(p_{\mathrm{B}}\right)=V_{\mathrm{B}^{\prime}}-V_{\mathrm{B}} \approx 8 \mathrm{~cm}^{3} \mathrm{STP} / \mathrm{g}$. The subloops CDC and $\mathrm{AB}^{\prime} \mathrm{A}$ are not congruent. The lack of congruence is equal to about $(29-8) \mathrm{cm}^{3} \mathrm{STP} / \mathrm{g}$.

roughness and modulation in the mesopore diameter along the channel. ${ }^{35}$ Thus, it is generally admitted that the pore walls of SBA-15 are more corrugated than that of MCM41 , the pores of which are not interconnected, and that the effect of constrictions in channels cannot be completely ruled out for SBA-15. ${ }^{36}$ However, if we compare the hysteresis loop shown in Figure 1 to that shown, for example, by Kruk and co-workers ${ }^{37}$ in their Figure 2, we cannot see any difference except the pressure at which they are observed. This shows that the SBA-15 under study is not significantly more corrugated than MCM- 41 .

The effect of more important defects, intentionally introduced in the mesopores of SBA-15, has been studied. It has been shown recently that, when SBA-15 silicas are formed in a synthesis mixture with TEOS/EO $\mathrm{EO}_{20} \mathrm{PO}_{70} \mathrm{EO}_{20}$ molar ratio higher than the usual value 59, a fraction of the mesopores exhibits porous plugs, constrictions and surface corrugation. ${ }^{38,39}$ Note that the value 59 for TEOS/ $\mathrm{EO}_{20} \mathrm{PO}_{70} \mathrm{EO}_{20}$ molar ratio was used for the first time by Zhao and co-workers ${ }^{24}$ and taken up again in many other studies and also by us in this work to form well ordered hexagonal mesoporous silica structure with uniform pore size. The presence of porous plugs and constrictions results in an appreciable widening and tailing of the hysteresis loops compared to standard SBA-15 silicas. ${ }^{38,39}$ This suggests that the standard SBA-15 silica studied in this paper does not show narrow constrictions which could lead to the formation of liquid bridges and gas bubbles into the mesopores during the condensation process and hence to pore blocking/network effects.

Let us now analyze the results obtained in other ordered porous materials: (1) MCM-41 and (2) $\mathrm{p}^{+}$-type porous silicon.

(1) $M C M-41$. it is well-known that there is no interconnection between the primary pores of MCM-41. Indeed, it has been shown that nanowires prepared by infiltration of the pores of MCM- 41 with carbon ${ }^{40}$ or platinum ${ }^{31}$ did not agglomerate, or when the agglomerates are observed,

(35) Liu, Z.; Terasaki, O.; Ohsuna, T.; Hiraga, K.; Shin, H. J.; Ryoo, R. ChemPhysChem 2001, 4, 229.

(36) Morishige, K.; Nakamura, Y. Langmuir 2004, 20, 4503.

(37) Kruk, M.; Jaroniec, M. Langmuir 1997, 13, 6267.

(38) Van Der Voort, P.; Ravikovitch, P. I.; De Jong, K. P.; Benjelloun, M.; Van Bavel, E.; Janssen, A. H.; Neimark, A. V.; Weckhuysen, B. M.; Vansan, E. F. J. Phys. Chem. B 2002, 106, 5873.

(39) Kruk, M.; Jaroniec, M.; Joo, S. H.; Ryoo, R. J. Phys. Chem. B 2003, 107, 2205.

(40) Ryoo, R.; Joo, S. H.; Kruk, M.; Jaroniec, M. Adv. Mater. 2001, 13,677 . 
they did not agglomerate in ordered bundles as is observed for SBA-15, the latter providing unequivocal evidence that the ordered mesopores in SBA-15 are interconnected. However, McNall and co-workers ${ }^{33}$ have obtained in MCM41 primary descending scanning curves similar to that shown in Figure 4 which suggests that in MCM-41, as is the case in SBA-15, the pores do not empty independently of one another. Therefore, this raises the question of pore blocking/network effects in MCM-41.

(2) $p^{+}$-Type Porous Silicon. Let us recall some properties of this porous material. ${ }^{20} \mathrm{p}^{+}$-type porous silicon, formed by anodic dissolution of highly boron doped (100) Si single crystal, is composed of tubular pores separated from one another by Si single-crystal walls of constant thickness. It has been shown that the pores are not interconnected and that the average direction of the pore axis is aligned to within $\pm 0.1^{\circ}$ with the [100] crystal axis. ${ }^{41}$ The pores, with polygonal cross-section of mesopore sizes, have a large size distribution but the same length proportional to the dissolution time. Once the chosen pore length is obtained, the anodic current can be cut off to form a porous layer supported by the Si substrate with pores closed at one end or can be increased to go into an electropolishing regime during which the $\mathrm{Si}$ walls are dissolved at the bottom of the pores which allows separation of the porous layer from the Si substrate to obtain a membrane with pores open at both ends. It is worth noting that such a process leaves the porous layer unchanged since the resistivity of the silicon walls which separate the pores is of the same order of magnitude as that of intrinsic compact silicon, ${ }^{42,43} \rho \approx 10^{5} \Omega \mathrm{cm}$, whereas the resistivity of the substrate is equal to $10^{-3} \Omega \mathrm{cm}$. The condensation branch was systematically observed at higher pressure for the membrane than for the corresponding porous layer with pores open at one end. This indicates that the filling of each pore closed at one end occurs from the bottom to the top without the formation of liquid bridges in narrow sections if any, otherwise the two boundary condensation branches should be superimposed. Studies of transmission electronic microscopy plan views ${ }^{20}$ indicate that a $2 \mathrm{D}$ image perpendicular to the pore axis yields porosity information consistent with that obtained by weighing, which corresponds to the 3D morphology.

In this porous material, we have observed large hysteresis loops of type H2. ${ }^{20}$ Moreover, the pores do not drain independently of one another as indicated by the asymmetry of the boundary hysteresis loop (type H2) ${ }^{20}$ and confirmed by experiments similar to those presented here. ${ }^{45}$ We have questioned pore blocking/network effects as being the cause of these results for two reasons: (a) if constrictions exist in such a porous material, they are randomly distributed within the pores and occupy a small

(41) Amsel, G.; d'Artemare, E.; Battistig, G.; Morazzani, V.; Ortega C. Nucl. Instr. Methods B 1997, 122, 99 .

(42) Ben Chorin, M. In Properties of Porous Silicon; Canham, L. Ed.; INSPEC Publishers: 1997; Chapter 6, p 165.

(43) Grosman, A.; Ortega, C. In Properties of Porous Silicon; Canham, L., Ed.; INSPEC Publishers: 1997; Chapter 11, p 328.

(44) Malanoski, A.; van Swol, F. Phys. Rev. E 2002, 66, 041603.

(45) Grosman, A.; Ortega, C. to be published. volume compared to the total pore volume. Therefore, the presence of one constriction at the top of each pore for the layer or at both ends of each pore for the membrane (whatever the membrane thickness is), as was proposed ${ }^{44}$ to explain the presence of a hysteresis loop of type $\mathrm{H} 2$ in this porous material, is very unlikely. If constrictions exist, they are not the cause of the observed hysteresis; (b) as we have already noted the pores are not interconnected and there is no constriction within the pores which could lead to the formation of liquid bridges during capillary condensation. Thus, at a given pressure along the condensation branch, a pore is either empty (with an adsorbed film on the pore walls) or filled without any bubble of gas inside. Hence, according to pore blocking/network effects, the pores should empty independently of one another which is not the case.

\section{Conclusion}

The first conclusion of this paper is that the pores in SBA-15, MCM-41, and $\mathrm{p}^{+}$-type porous silicon do not empty independently of one another. The pressure at which a mesopore empties depends on the state of the neighboring pores and hence on the history of the system as a whole. None of the experimental results cited above, related to the morphology of MCM- $41^{31,40}$ and $\mathrm{p}^{+}$-type porous silicon, ${ }^{20}$ have shown the presence of interconnections between the pores. In SBA-15, the experiments ${ }^{31}$ do not reveal the presence of intrawall pores in the mesopore range but only reveal the presence of micropores which are filled with gas at pressures at which the hysteresis loops are observed. Consequently, we question pore blocking/network effects as being the mechanism by which the pores interact in these ordered porous media.

Wallacher and co-workers, ${ }^{21}$ in a recent paper, have also questioned pore blocking as being the cause of the hysteresis loop observed in $\mathrm{p}^{+}$-type porous silicon and think that the evaporation proceeds through metastable states.

The second conclusion is that, in these ordered porous materials, the boundary evaporation branch does not represent thermodynamic equilibrium transitions for the pores, otherwise they should empty independently of one another which is not the case.

Recently, Morishige and co-workers, ${ }^{36}$ by analyzing the temperature dependence of capillary condensation and evaporation for nitrogen in MCM-41 and in SBA-15, also came to the conclusion that capillary evaporation does not take place near the equilibrium in these systems.

We think, with the groups cited above, ${ }^{21,36}$ that the questions raised by the condensation-evaporation processes in porous media are not yet solved, and that further experiments and calculations are needed to elucidate these processes and in particular, to explain (i) the presence of liquidlike metastable states and (ii) the mechanism by which the pores interact during the evaporation process in ordered porous materials.

Acknowledgment. The authors thank D. Morineau and co-workers for providing the SBA-15 samples.

LA0510300 